\title{
Mimosa bahamensis (Leguminosae) en la Península de Yucatán, México
}

\author{
Rosaura Grether y SARA L. CAMARGo-RICALDE ${ }^{1}$
}

\begin{abstract}
RESUMEN. Este trabajo presenta la historia nomenclatural de Mimosa bahamensis Bentham, conocida comúnmente como "saskatzim" o "sak-katzim", el estudio de su fenología y de su distribución en la parte mexicana de la Península de Yucatán, como un elemento importante en áreas taladas de la selva, y en tintales y en vegetación de bajos. Se destaca su abundancia en terrenos de cultivo sujetos a quemas periódicas o abandonados y a la orilla de caminos y veredas. Se discute el carácter oportunista y típicamente secundario de esta especie, así como las posibles causas de su distribución disyunta.
\end{abstract}

ABSTRACT. This paper includes the nomenclature of Mimosa bahamensis Bentham, commonly known as "saskatzim" or "sak-katzim", its phenology and its distribution range in the Mexican part of the Yucatan Peninsula, as an important element, mainly in disturbed areas of forests, "tintales" and shrub vegetation. Its presence in cultivated or abandoned lands under periodic fires, and along roads and trails is emphazised. The invasive and typycally secondary character of this species, as well as the possible causes of its disjunct distribution are discussed.

Este trabajo surge de manera colateral al estudio taxonómico del género Mimosa dentro del proyecto interinstitucional Flora Mesoamericana, particularmente en lo que se refiere a la participación de la UAM-Iztapalapa.

Mimosa bahamensis es una especie conocida de la Península de Yucatán, incluyendo Guatemala y Belice, y de las Islas Bahamas. El objetivo de este trabajo es delimitar el área de distribución de la especie en la parte mexicana de la Península de Yucatán, establecer los tipos de vegetación y condiciones en que crece, así como determinar su fenología.

${ }^{1}$ Departamento de Biología, División de C.B.S., Universidad Autónoma Metropolitana-Iztapalapa. Apdo. Postal 55-535, CP 09340, México, D.F. 


\section{Metodología}

Se llevó a cabo la revisión de ejemplares en herbarios nacionales: UAMIZ y MEXU, y del extranjero: GH, K, MO y US, y se realizaron excursiones a la Península de Yucatán durante un período de cinco años, para recolectar material biológico; así como para llevar a cabo observaciones periódicas, de tipo directo, sobre la fenología de $M$. bahamensis, para delimitar su área de distribución y determinar los distintos tipos de vegetación y condiciones en que crece.

Para determinar las características del suelo en sitios en los que se encuentra esta especie, se colectaron, con base en un muestreo al azar, cinco muestras, a 20-30 cm de profundidad en las siguientes localidades: $14.5 \mathrm{~km}$ al E de Cafetal, camino a Uvero, al $\mathrm{N}$ de la Laguna de Bacalar, Quintana Roo; $1.5 \mathrm{~km}$ al N de Xel-ha, Quintana Roo; $13 \mathrm{~km}$ al W de Valladolid, Yucatán; 1 km al W de Kopomá, km 143 carretera de Campeche a Mérida, Yucatán; y 23 km al NW de Yohaltún, al SE de Champotón, Campeche. Los análisis físico-químicos fueron realizados por la Comisión de Estudios del Territorio Nacional.

Para analizar las posibles causas de la distribución disyunta de $M$. bahamensis, se consideró la posibilidad de dispersión de las semillas por medio de las corrientes marinas (Gunn y Dennis, 1976) y se llevaron a cabo pruebas de flotabilidad de frutos en agua marina. Asimismọ, se realizó la revisión bibliográfica de la dirección de los ciclones en el Caribe en diferentes épocas del año (Anónimo, 1970).

\section{RESULTADOS}

\section{Historia Nomenclatural}

Mimosa bahamensis fue descrita por Bentham en 1842 y considerada por mucho tiempo como una especie endémica de las Islas Bahamas. Por otra parte, Rose y Robinson describieron Mimosa hemiendyta en 1903, la cual ha sido citada por diversos autores como endémica de la Península de Yucatán.

Britton en 1928, transfirió estas dos especies a un nuevo género Pteromimosa, al que caracterizó por presentar flores en cabezuela, corola 4-5-lobulada, estambres 8-10 y fruto dividido en artejos con margen angostamente lacerado-alado; el autor distinguió Pteromimosa bahamensis (Benth.) Britton, por el fruto sésil o casi sésil, los folíolos glabros y la corola 4-lobulada, y Pteromimosa hemiendyta (Rose \& Rob.) Britton, por el fruto largamente estipitado, los folíolos pubescentes cuando jóvenes y la corola de 4-5-lobulada.

Pteromimosa no se reconoce como un género válido, ya que sus caracteres coinciden con los del género Mimosa. Muchas especies de Mimosa presentan flores dispuestas en cabezuelas, el número de lóbulos de la corola puede variar de 4 a 5 , aún en una misma especie, y el fruto dividido en artejos es característico de la mayoría de las especies; por 
otra parte, el margen lacerado-alado se presenta también en Mimosa lacerata Rose, aunque en este caso, el fruto no está dividido en artejos.

Rudd (1969), realizó una revisión cuidadosa de los ejemplares de Mimosa hemiendyta colectados en la Península de Yucatán y de los ejemplares de $M$. bahamensis de las Islas Bahamas, llegando a la conclusión de que se trata de una misma especie: Mimosa bahamensis Benth., con distribución disyunta: Península de Yucatán-Islas Bahamas.

La revisión de los ejemplares tipo de Mimosa bahamensis y de Mimosa hemiendyta, así como de diversos ejemplares de las Islas Bahamas y las observaciones de campo en la Península de Yucatán, nos hacen coincidir con la opinión de Rudd (1969). Por lo anterior, se reconoce una sola especie, cuyo nombre correcto es Mimosa bahamensis Benth., con la siguiente sinonimia:

Mimosa bahamensis Benth., J. Bot. (Hooker) 4: 408 (1842). Tipo: Tropical America, Bahama Islands, (Herb. Hooker) (Holotipo: K! foto MEXU! ex K).

Pteromimosa bahamensis (Benth.) Britton, N. Amer. Fl. 23: 171 (1928).

Pteromimosa hemiendyta (Rose et Robinson) Britton, N. Amer. Fl. 23: 172 (1928). Mimosa hemiendyta Rose et Robinson, Contr. U. S. Natl. Herb. 8: 32 (1903). Tipo: México, Campeche, Apagota, near Yohaltún, 31 Dec. 1900, Goldman 513 (Holotipo: US!; fragmentos: GH!).

Esta especie presenta variación en el número de lóbulos de la corola (4-5) y en el número de estambres (8-10); los frutos varían de sésiles a estipitados aún en un mismo individuo, son generalmente pubescentes y en ocasiones setosos; sin embargo, no se ha encontrado relación de estas variaciones con un área geográfica delimitada, por lo que no es posible distinguir taxa subespecíficos.

Rasgos Fisiográficos y Climáticos de la Península de Yucatán

La Península de Yucatán comprende los estados de Yucatán, Campeche y Quintana Roo en México, Belice y la región del Petén en Guatemala (fig. 1).

Es una gran planicie formada por rocas calizas de origen Terciario y Cuaternario. En la parte mexicana de la Península, las elevaciones son escasas, destacando la Sierrita de Ticul y la Meseta de Zohlaguna con altitudes no mayores de $400 \mathrm{msnm}$.

Una de las características fisiográficas más notables de esta zona, es la ausencia de corrientes superficiales, excepto en el extremo sureste donde se encuentra el Río Hondo y en el extremo suroeste, donde corren los cauces de los ríos Champotón, San Pedro y Candelaria. Existen pocas corrientes de temporal y tan pronto como las lluvias cesan, el agua se filtra rápidamente contribuyendo a formar los cenotes (Miranda, 1964).

Los suelos de la mayor parte de la Península son de tipo calomórfico, como las rendzinas negras predominantes y las rendzinas rojas; estos suelos tienen un drenaje muy eficiente. En algunas partes se presentan suelos de tipo hidromórfico, como los suelos de gley (ak'alché) que son más compactos y con drenaje deficiente, que provoca la acumulación de agua cuando menos durante una época del año.

Las muestras de suelos colectadas dentro del área de distribución de M. bahamensis presentan las siguientes características: 


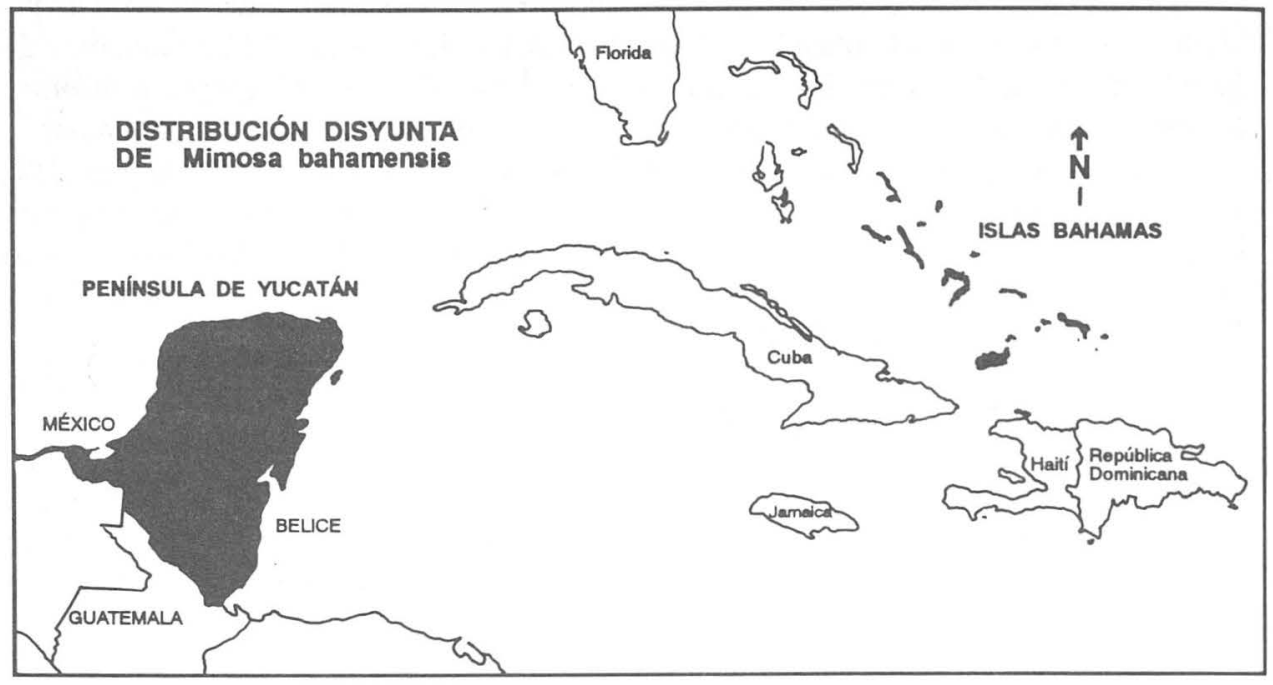

Fig. 1. Distribución disyunta de Mimosa bahamensis en la Península de Yucatán y en las Islas Bahamas.

El color en seco varía del pardo grisáceo muy oscuro al gris muy oscuro hasta el gris; en húmedo, varía del negro al pardo muy oscuro, hasta el gris muy oscuro.

Estos suelos presentan una textura media, desde migajones arenosos, hasta migajones arcillosos; y sólo en un sitio de muestreo (Yohaltún, Campeche), se presenta una textura arcillosa.

$\mathrm{El} \mathrm{pH}$ es ligeramente alcalino (7.54 en promedio), por lo que se consideran suelos muy ricos en materia orgánica. Las concentraciones de cationes extractables son bajas, principalmente del Sodio ( 0.1 a $0.5 \mathrm{meq} / 100 \mathrm{gr}$ ) y del Potasio ( 0.3 a $0.9 \mathrm{meq} / 100 \mathrm{gr}$ ); no obstante, las concentraciones de Calcio (37.1 a $44.8 \mathrm{meq} / 100 \mathrm{gr}$ ) y de Magnesio (2.1 a $5.2 \mathrm{meq} / 100 \mathrm{gr}$ ) son altas, por lo que el porcentaje de saturación de bases (80 a $95 \%$ ) y la capacidad de intercambio catiónico total (45.1 a $57.2 \mathrm{meq} / 100 \mathrm{gr})$ son altos. Sin embargo, el fósforo extraíble (1.1 a 2.8 ppm) es muy bajo.

Las características analizadas indican que estos suelos (fig. 2) son fértiles, con alto contenido de materia orgánica y, por lo tanto, alta retención de humedad. Presentan pH 
ligeramente alcalino, con alta capacidad de intercambio catiónico total y son pobres en fósforo.

En la mayor parte de la Península, el clima es cálido-subhúmedo (Aw de Köppen) con varios grados de humedad y temperatura media mensual entre 20.5 y $30.5 \mathrm{C}$. La precipitación media anual más alta, alrededor de $2000 \mathrm{~mm}$, se presenta en la región sureste, disminuyendo hacia el noroeste, donde el clima es seco (B de Köppen), la precipitación media anual disminuye, no siendo mayor de $500 \mathrm{~mm}$ (Quero, 1981).

Las variantes climáticas de la Península aparentemente no influyen en la distribución de $M$. bahamensis, aunque si tienen efecto sobre su fenología.

Fenología

La información fenológica de especies del género Mimosa es muy escasa. Los únicos antecedentes que se tienen, son el resultado de las observaciones periódicas a lo largo de 3 años, llevadas a cabo por Grether (1982), para determinar las épocas de foliación, floración y fructificación de $M$. biuncifera Benth. y de $M$. monancistra Benth. en la Cuenca Alta del río La Laja, Guanajuato.

En el caso de M. bahamensis, a partir de observaciones periódicas, de tipo directo, realizadas y registradas durante cinco años en muy diversas localidades de la Península de Yucatán (fig. 2), se determinó la fenología (fig. 3) y la dispersión de esta especie.

Foliación: $M$. bahamensis es una especie caducifolia, cuya foliación empieza generalmente en el mes de abril; en esta época es frecuente observar poblaciones en las que la mayoría de los individuos presentan gran cantidad de brotes y renuevos de hojas. Durante los meses de mayo y junio, el follaje se encuentra bien desarrollado en la mayoría

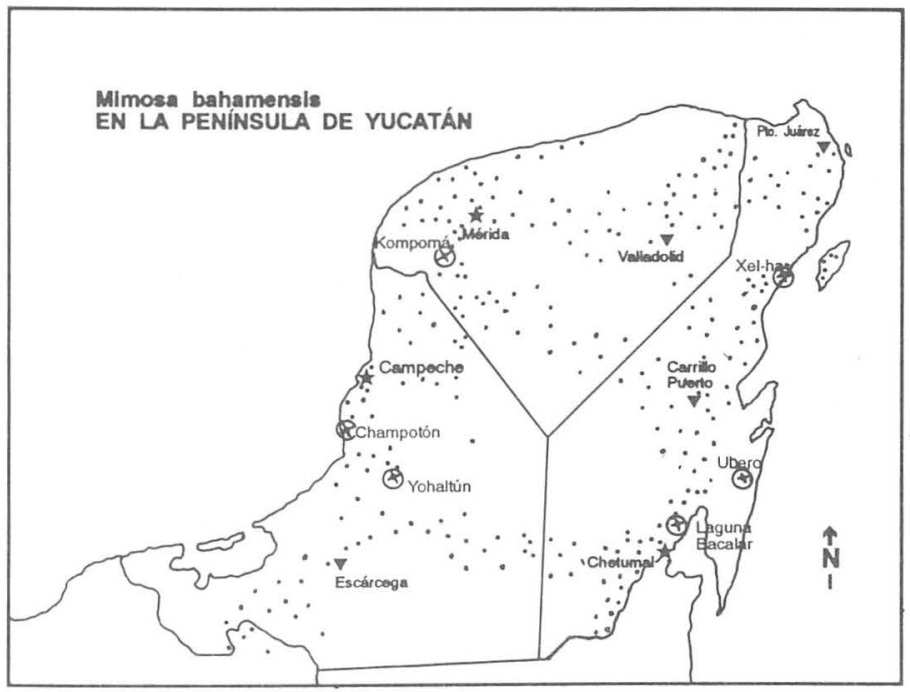

Fig. 2. Distribución de Mimosa bahamensis en la parte mexicana dẹ la Península de Yucatán. 


\section{FENOLOGÍA DE Mimosa bahamensis}

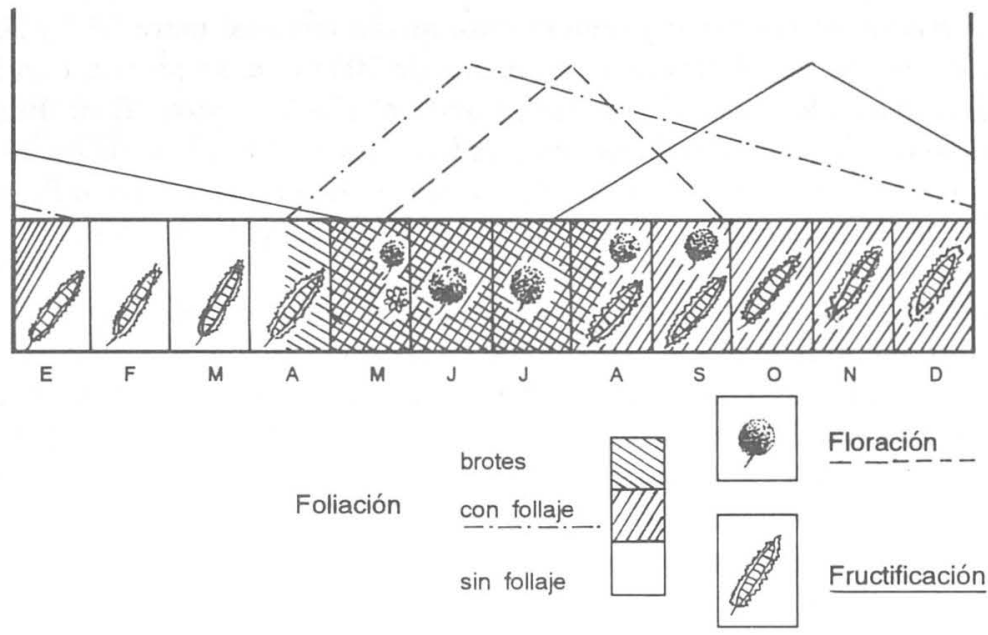

Fig. 3. Fenología de Mimosa bahamensis. Epocas de foliación, floración y fructificación en la parte mexicana de la Península de Yucatán. Los picos de las líneas únicamente señalan los meses de máxima foliación, floración y fructificación.

de las poblaciones y persiste hasta el mes de noviembre; las hojas maduras empiezan a caer en los meses de diciembre y enero, y es frecuente observar las ramas blanquecinas sin follaje en los meses de febrero y marzo (fig. 4 y 5 ).

Floración: La floración se inicia a fines de mayo en la mayor parte de la Península, en el mes de junio es frecuente observar gran cantidad de botones y algunas cabezuelas abiertas; durante los meses de julio a septiembre, esta especie se encuentra en plena floración y se distingue fácilmente por la gran cantidad de flores rosadas, dispuestas en grandes panículas de cabezuelas; el período de floración termina a fines de septiembre en la mayor parte de la Península (fig. 5).

Fructificación: Los frutos inmaduros se observan desde el mes de julio y los frutos maduros son muy abundantes durante los meses de septiembre y octubre. Cada individuo produce gran cantidad de frutos maduros con semillas bien desarrolladas, aunque se ha observado el ataque de brúquidos en algunas poblaciones; una buena cantidad de frutos persisten en la planta, hasta los meses de abril o mayo del año siguiente. Su dispersión es paulatina a lo largo de un período de 8 a 9 meses (fig. 6). 


\section{MIMOSA BAHAMENSIS EN LA PENINSULA DE YUCATAN}

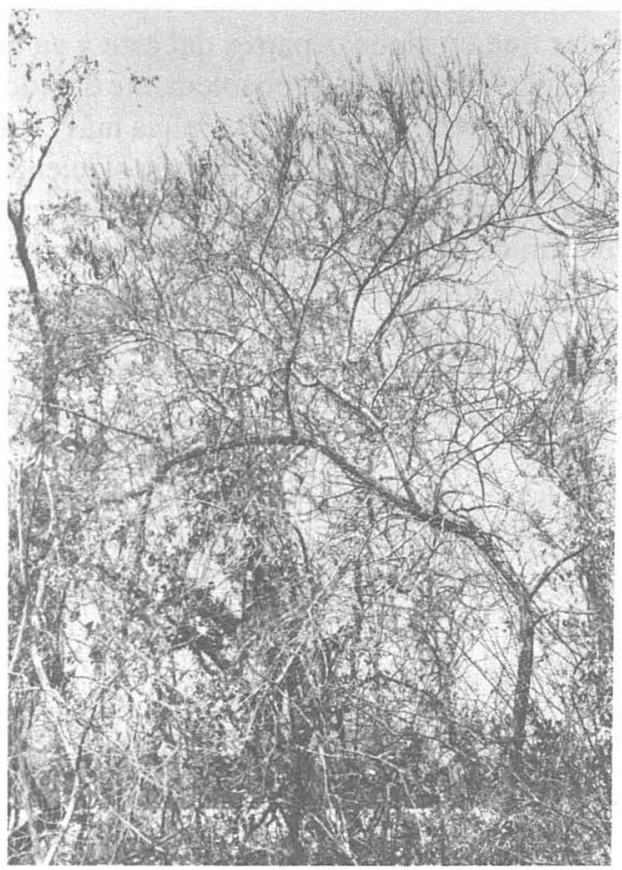

Fig. 4. Arbol de Mimosa bahamensis sin follaje, en terreno de cultivo abandonado.

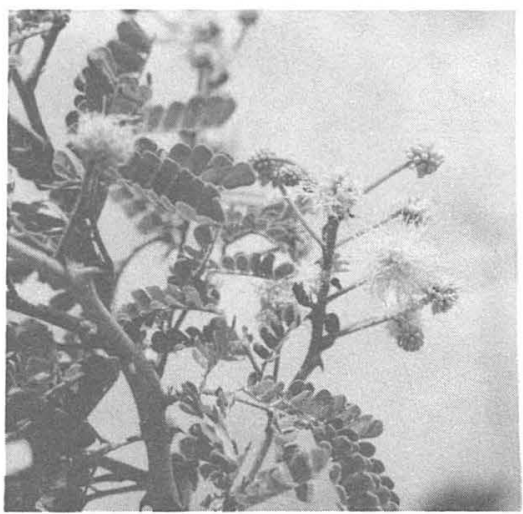

Fig. 5. Mimosa bahamensis en época de floración. Se observan las cabezuelas arregladas en panículas y las hojas maduras. 
Es importante señalar que en algunas partes del este y en el sureste de Quintana Roo, la fenología de esta especie se retrasa, probablemente debido al clima más húmedo, con alta precipitación. En esta zona, la foliación se inicia más tarde y se observan restos de hojas del año anterior, aún en el mes de abril del año siguiente; la floración se inicia generalmente hasta el mes de agosto, las poblaciones se encuentran en plena floración en octubre y su período termina hasta noviembre o principios de diciembre, de manera que los frutos maduros son abundantes en los meses de noviembre a enero del año siguiente. El comportamiento fenológico de esta especie es cambiante, dependiendo de la variación de las condiciones ambientales año tras año durante períodos prolongados.

Dispersión: La dispersión de frutos y semillas se lleva a cabo tanto en forma natural, como por intervención humana.

En forma natural, los frutos son diseminados por el viento que produce el roce de unas ramas contra otras y los frutos maduros y secos se desprenden fácilmente. Dado que presentan un margen alado persistente, observamos que son desplazados por el

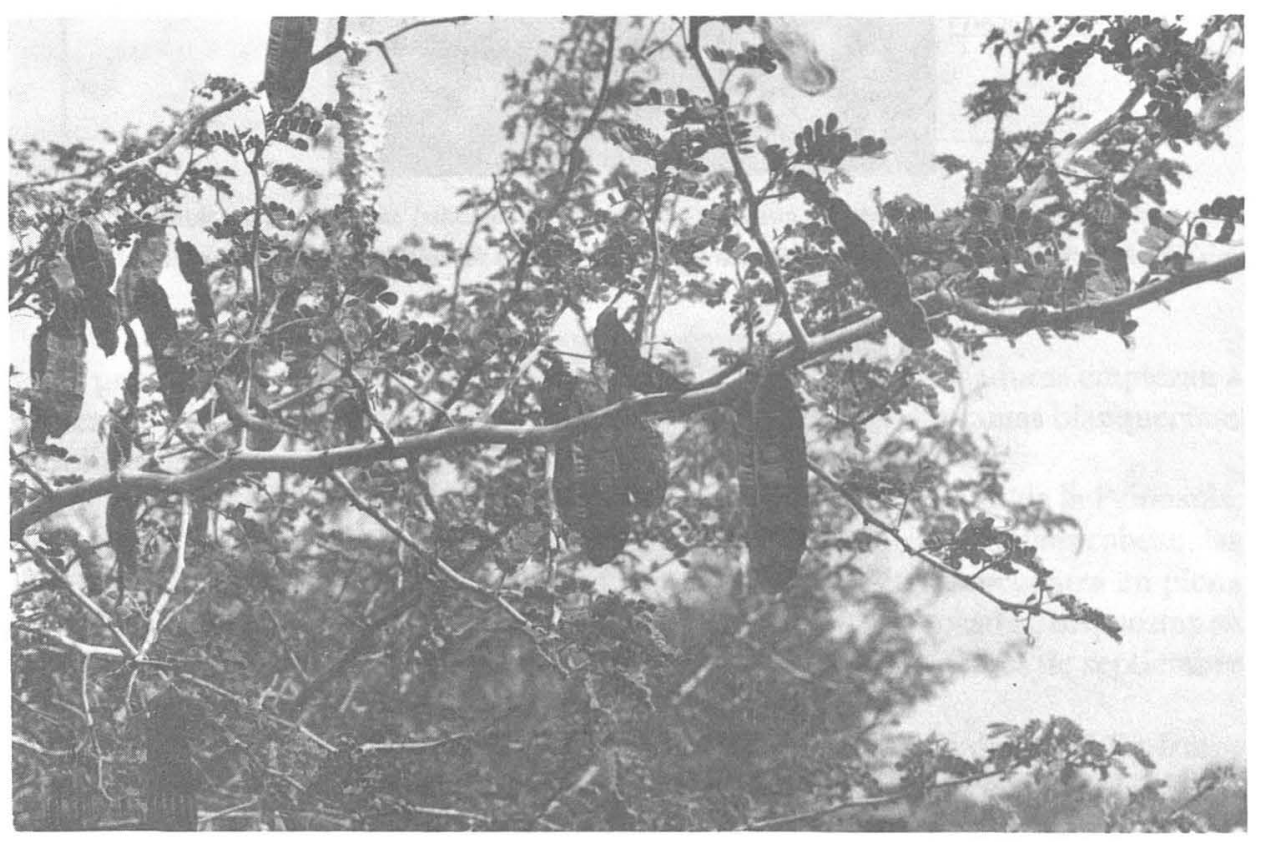

Fig. 6. Frutos maduros de Mimosa bahamensis. Se observan los artejos y el margen lacerado-alado. 
viento en un radio de 5 a $6 \mathrm{~m}$ alrededor de la planta madre, en comunidades más o menos densas, mientras que llegan hasta 8 ó $10 \mathrm{~m}$ de la planta madre, en áreas abiertas.

El fruto presenta de 5 a 9 artejos, cada uno con una semilla, éstos se separan fácilmente del margen alado durante la diseminación o ya estando sobre el suelo; la producción de una gran cantidad de frutos y semillas por individuo (observación cualitativa) y la diseminación paulatina de los mismos durante un período de 8 a 9 meses favorece notablemente la invasión de nuevas áreas.

Por otra parte, se llevaron a cabo pruebas de flotabilidad en agua marina, y se observó que los frutos completos y aún los artejos aislados, se sumerjen fácilmente después de 24 a 72 hrs., por lo que no es probable su diseminación por medio de las corrientes marinas.

En época de ciclones (agosto-octubre) los vientos son más fuertes y los frutos pueden ser transportados a distancias mayores e invadir nuevas áreas perturbadas; al mismo tiempo, la lluvia es otro factor que contribuye al desprendimiento de los artejos y, por tanto, a la dispersión de semillas aisladas.

Por su parte, el hombre contribuye a la diseminación artificial de los frutos y a la apertura de espacios favorables para el establecimiento de nuevos individuos, al rozar y talar las selvas, al hacer nuevos caminos y al limpiar los terrenos de cultivo. Los frutos maduros y secos se desprenden muy fácilmente de la planta y liberan una gran cantidad de semillas al ser removidos directa o indirectamente por el hombre, en el desempeño de estas actividades.

\section{Distribución Geográfica}

La distribución de $M$. bahamensis en la parte mexicana de la Península de Yucatán se determinó a partir del trabajo de campo, de la colecta de ejemplares botánicos y de la revisión de ejemplares de herbario.

Mimosa bahamensis tiene una amplia distribución en la Península de Yucatán y en las Islas Bahamas (fig. 1). En la parte mexicana de la Península se conoce comúnmente como "saskatzim" o "sak-katzim", que significa el katzim blanco o falso, ya que la especie conocida como el verdadero katzim es Acacia gaumeri (Barrera et al., 1976). Su límite de distribución en México está dado por las planicies aluviales de Tabasco y del norte de Chiapas y hacia el sur, se extiende hasta la región del Petén en Guatemala y hasta el centro de Belice (fig. 2).

Hacia las costas de la Península, su distribución está limitada por las dunas, los manglares y las lagunas costeras, principalmente en la costa norte de Yucatán; se extiende generalmente hasta $3 \mathrm{~km}$ de la línea de costa, aunque en algunos casos se han observado poblaciones a $500 \mathrm{~m}$ del litoral, en sitios donde se forman salientes y ensenadas rocosas, como sucede en los alrededores de Seybaplaya, en el estado de Campeche y en la región de Xel-ha en Quintana Roo.

Se encuentra en áreas sujetas a disturbio de toda la parte mexicana de la Península, siendo más abundante en el norte y centro, probablemente debido a que esta zona ha sido perturbada durante un período más prolongado que la región del sur. 


\section{Mimosa bahamensis una especie oportunista}

De acuerdo al concepto de Thompson (1991), M. bahamensis puede considerarse como una especie oportunista, ya que aparece en sitios perturbados abiertos, y va colonizando nuevas áreas, dentro de un rango amplio de condiciones ambientales y, además, puede convertirse en uno de los componentes dominantes del habitat que ocupa.

Por lo anterior, M. bahamensis es una especie típicamente secundaria y frecuentemente se encuentra como un elemento abundante en: a) áreas taladas de las selvas, b) en tintales y bajos alterados, c) en terrenos de cultivo sujetos a quemas periódicas o abandonados y d) a la orilla de los caminos y veredas.

a) Areas taladas dentro de las selvas.

Mimosa bahamensis invade las áreas taladas de las selvas desarrollándose rápidamente, al principio como un arbusto y en algunos años como un árbol que alcanza alturas no mayores de $8 \mathrm{~m}$. Esta especie es uno de los elementos más abundantes de las comunidades secundarias resultantes de la tala, en las que muchas veces predominan las leguminosas.

Forma parte importante de la vegetación alterada de las selvas bajas y medianas deciduas del noroeste, oeste y centro del estado de Yucatán y del norte de Campeche, donde se asocia frecuentemente con otras leguminosas (listado 1).

Por otra parte, destaca por su abundancia en los claros y áreas taladas de la selva baja subdecidua con Pseudophoenix, Coccothrinax y Thrinax, que se presenta en los alrededores de la caleta de Xel-ha, en Quintana Roo (Quero, 1981); éste es uno de los sitios más cercanos a la costa, donde se ha encontrado, aproximadamente a $500 \mathrm{~m}$ del litoral, asociada tanto con especies secundarias, como con algunos de los elementos primarios de esta selva (listado 2).

En los alrededores de Ubero, Quintana Roo, también se encuentra a $500 \mathrm{~m}$ de la línea de costa, asociada con Coccoloba en áreas perturbadas de selva baja subdecidua con Coccothrinax.

En el norte de la Isla de Cozumel, se asocia con Bursera simaruba (L.) Sarg., Caesalpinia violacea (Mill.) Standl., Lysiloma latisiliqua (L.) Benth., Metopium brownei (Jacq.) Urban y Zapoteca formosa (Kunth) H. Hern. subsp.formosa; se presenta en áreas taladas de selva baja subdecidua. Es interesante mencionar que esta especie, no se encuentra en Isla Mujeres, dado que esta pequeña isla está sujeta a una gran influencia marina en toda su extensión y los suelos son arenosos, predominando la vegetación de dunas, así como los manglares en ciertas partes.

Se presenta en forma abundante en los claros y sitios alterados de la selva de transición entre baja y mediana subdecidua al noroeste y sureste de Felipe Carrillo Puerto, en Quintana Roo, asociada con otras especies (listado 3).

Mimosa bahamensis invade las áreas perturbadas de las selvas altas de la Península. Entre Colonia Yucatán y El Cuyo, en el noreste del estado de Yucatán, es frecuente 
encontrar acahuales viejos de selva alta subperennifolia con Manilkara y Thrinax (listado 4).

Se ha observado la abundancia de esta especie en las partes quemadas y taladas de la selva alta subperennifolia con Manilkara, Thrinax y Coccothrinax que se encuentra al sur de Cancún, Quintana Roo (listado 5).

El área ocupada por esta selva está siendo destruída rápidamente por encontrarse cerca del complejo turístico de Cancún, actualmente se obtiene de esta zona, material para construcción y relleno de terrenos inundables. $M$. bahamensis es una de las pocas especies que logran desarrollarse aún en las partes donde se ha eliminado no sólo el suelo, sino también parte de la roca caliza, por lo que desempeña un papel importante para evitar una mayor erosión y contribuir a la restitución del suelo a largo plazo.

Hacia el sur de la Península, donde la vegetación dominante es la selva alta subperennifolia con Manilkara, Bucida y Cryosophila, es frecuente encontrar áreas perturbadas en las que destaca $M$. bahamensis. En la meseta de Zohlaguna, así como en los alrededores de Yohaltún, Campeche, esta selva ha sido explotada durante mucho tiempo para la obtención de madera, principalmente caoba y actualmente se encuentra muy alterada. $M$. bahamensis es uno de los elementos secundarios más importantes (listado 6).

En las selvas del sur de Quintana Roo, M. bahamensis es más escasa y se asocia con otras especies, además de las ya mencionadas (listado 7).

b) Tintales y vegetación de bajos alterados.

El tintal es una selva baja, a veces mediana, subdecidua, de 8 a $12 \mathrm{~m}$ de alto, caracterizada por la predominancia de Haematoxylon campechianum L.; este tipo de vegetación es característico de los suelos de ak'alché, que son planos y profundos con drenaje deficiente y que se inundan periódicamente (Miranda, 1964).

Encontramos tintales cerca de la costa norte de Yucatán, en el centro y sur de Campeche y en la parte occidental de Quintana Roo; si bien M. bahamensis no forma parte del tintal primario, si invade los tintales alterados (Barttlet, 1936; Lundell, 1937), sobre todo en la parte periférica más seca de estas comunidades, donde crece como árbol o arbusto hasta 6 ó $7 \mathrm{~m}$ de alto, abundante y con ramas muy largas, generalmente recargadas sobre otros árboles y arbustos (listado 8).

Los bajos extensos del centro de Campeche y este de Quintana Roo, se caracterizan por una asociación de Cameraria-Haematoxylon-Metopium, que constituye una selva muy baja, con aspecto de matorral alto en ocasiones, donde la vegetación herbácea es escasa debido a las frecuentes inundaciones (Miranda, 1964); M. bahamensis invade también estas áreas, donde el suelo tiene altas concentraciones de carbonato de calcio, y forma matorrales densos sobre todo en el bajo de Acatuch, al oeste de la laguna de Bacalar, Quintana Roo, particularmente en franjas anchas de suelo removido a los lados de la carretera o bien se asocia con otras especies (listado 9). 
Además, esta especie crece en las orillas del tasistal, caracterizado por la predominancia de Acoelorraphe wrightii Beccari, típico de zonas pantanosas.

c) Terrenos de cultivo.

Históricamente el sistema de roza-tumba-quema ha predominado en la agricultura de la Península de Yucatán. Los campesinos llevan a cabo el desmonte, dejando tocones de 0.5 a $1 \mathrm{~m}$ de alto; las raíces de árboles y arbustos no se destruyen y como consecuencia gran parte de la regeneración natural en estos terrenos consiste de los brotes de los árboles y arbustos originales. Por esta razón, la composición de las selvas en disturbio depende de la composición existente antes del desmonte y de la frecuencia de los disturbios (Hernández X., 1962).

El cultivo de henequén es el más importante de la región semiárida del noroeste de la Península, principalmente del estado de Yucatán y parte de Campeche. Al final del período de aprovechamiento del henequén, en muchas ocasiones el terreno se deja en barbecho durante varios años antes de volverse a plantar. En este tiempo, la selva secundaria se desarrolla con una notable abundancia de leguminosas, entre las cuales destaca M. bahamensis (Staggerda, 1941; Sousa-Novelo, 1945).

Si bien, esta especie no forma parte de la vegetación primaria original, debe hacerse notar que la práctica del sistema de roza-tumba-quema durante un largo período, ha provocado que esta especie, oportunista y típicamente secundaria, sea uno de los elementos principales en la regeneración de la vegetación de terrenos en barbecho, ya que sus numerosos frutos y semillas se diseminan fácilmente.

Por otra parte, el cultivo de mayor importancia alimenticia en la Península es el maíz; los terrenos abandonados en barbecho se caracterizan también por la predominancia de leguminosas entre las cuales se encuentra casi siempre $M$. bahamensis, asociada con otras especies (listado 10) (Staggerda, 1941; Sousa-Novelo, 1945). En ocasiones, las primeras fases de la vegetación secundaria consisten de una asociación muy densa y casi pura de M. bahamensis.

Esta especie es resistente al fuego y retoña fácilmente de los tocones y aún de raíces parcialmente quemadas, desarrollándose rápidamente (fig. 7); si el terreno se abandona por un largo período, los individuos de esta especie crecen como árboles de hasta 6 ó 7 m de alto (fig. 4). Lundell (1934), destaca su abundancia en potreros derivados de tintales y posteriormente abandonados por 17 años, en el sur de Campeche.

d) Orilla de caminos y veredas.

Mimosa bahamensis, también se desarrolla en las áreas abiertas a la orilla de los caminos y veredas en casi toda la Península; ésto se debe a su fácil y rápida regeneración a partir de los tocones, aún cuando se hayan quemado parcialmente, así como a su propagación a partir de raíces parcialmente expuestas en el suelo removido a la orilla de caminos recién abiertos; en ocasiones forma matorrales casi puros, muy densos, bordeando los caminos; estos matorrales destacan fácilmente, sobre todo durante las épocas de floración y fructificación, aunque también se distinguen en la época de 




Fig. 7. Propagación vegetativa de Mimosa bahamensis a partir de un tocón y raíces parcialmente quemadas.

invierno por la red intrincada de ramas espinosas blanquecinas. Con frecuencia se asocia con otras especies que crecen a la orilla de los caminos (listado 11).

\section{DISCUSIÓN}

Consideramos que la amplia distribución y abundancia de esta especie, como uno de los elementos importantes de la vegetación secundaria de la Península de Yucatán, es el resultado, por una parte, de algunas características propias de la planta y, por otra, de varios factores externos que favorecen su desarrollo en áreas sujetas a disturbio.

Entre las características propias de esta especie podemos señalar las siguientes: a) la gran cantidad de frutos y semillas producidos por individuo cada año; b) el período relativamente largo de 8 a 9 meses de producción de frutos y dispersión paulatina de los mismos; c) el márgen lacerado-alado de los frutos, que permite una diseminación natural 
eficiente por el viento; y d) su resistencia al fuego y la facilidad con que se restituyen los individuos a partir de tocones, así como su propagación por el desarrollo de brotes a partir de ramas y de raíces expuestas y aún parcialmente quemadas.

Existen por otra parte, diversos factores externos, debidos a las actividades humanas, que crean nuevas condiciones favorables para su invasión y desarrollo. Entre los más importantes podemos mencionar: a) la tala de las selvas para obtención de maderas; b) la práctica del sistema de roza-tumba-quema en la mayoría de las zonas de cultivo de la Península. Este sistema en sus primeras fases de roza y tumba, deja áreas abiertas sujetas a la colonización por especies secundarias; las quemas, por otra parte, enriquecen el suelo en materia orgánica y descomponen algunos elementos, eliminan posibles especies competidoras, matan microorganismos del suelo, insectos, etc.; de manera que los campos de cultivo constituyen un medio muy favorable para el desarrollo de $M$. bahamensis, entre otras especies secundarias; c) la apertura de caminos y veredas favorece la diseminación de frutos y semillas, así como a la propagación vegetativa en el suelo removido y d) todas las actividades humanas mencionadas contribuyen a abrir espacios para el establecimiento de nuevos individuos.

Todas estas características sugieren el carácter oportunista y típicamente secundario de esta especie, cuya plasticidad le permite colonizar sitios perturbados.

Si bien, $M$. bahamensis no es una planta de gran importancia económica, aunque es utilizada para curtir pieles por la gran cantidad de taninos que contiene (Téllez y Sousa, 1982) y ha sido mencionada entre las especies melíferas de la Península Souza-Novelo et al., 1981), pero, su abundancia en esta región debe valorarse desde el punto de vista ecológico. Su capacidad de invadir nuevas áreas perturbadas, contribuye de manera importante a controlar la erosión y a restituir el suelo a largo plazo, aportando materia orgánica dado su caracter caducifolio y aportando nuevos individuos a través de la germinación de sus semillas y de su propagación vegetativa.

El conocimiento del papel de las especies secundarias, no sólo de la Península de Yucatán, sino en general de las regiones tropicales de México es fundamental, si consideramos que la vegetación primaria está siendo destruída en forma acelerada.

En lo que se refiere a la distribución de $M$. bahamensis en México, consideramos que las planicies aluviales de Tabasco y del norte de Chiapas, constituídas en gran parte por extensas zonas pantanosas, actúan como una barrera, debido a que esta especie no puede vivir en terrenos permanentemente inundados, ni en suelos aluviales ácidos, ya que se desarrolla fundamentalmente en substratos calizos y suelos con $\mathrm{pH}$ alcalino o neutro; sin embargo, se ha colectado en acahuales y potreros, así como a la orilla de áreas pantanosas en el Municipio de Balacán, Tabasco, cerca del límite con el estado de Campeche.

Respecto a las posibles causas de su distribución disyunta, en la Península de Yucatán y en las Islas Bahamas (fig. 1), Rudd (1969) señala que pudiera ser el resultado del transporte humano en un barco, ya sea que la planta fuera colectada intencional o 
accidentalmente y sugiere que la dirección del transporte fue de Yucatán a las Bahamas. Por otra parte, en este trabajo, se han analizado diversos factores que en un momento dado pudieran actuar como mecanismos de dispersión y explicar la distribución disyunta de esta especie:

La dirección de las corrientes marinas durante el verano en el Caribe (Gunn y Dennis, 1976), sugiere la posibilidad de dispersión de la Península de Yucatán a las Islas Bahamas. Tomando en cuenta que en algunos sitios esta especie crece muy cerca de la línea de costa, sus frutos alados podrían llegar al mar por medio del viento, ciclones y vientos huracanados; sin embargo, a partir de las pruebas de flotabilidad en agua marina, demostramos que los frutos completos y aún los artejos aislados se sumergen fácilmente después de 24 a $72 \mathrm{hrs}$., por lo que se descarta esta posibilidad.

Por otra parte, se revisó la dirección de los ciclones en el Caribe, en diferentes épocas del año (Anónimo, 1970); los ciclones van del S-SW al N-NE durante los meses de septiembre a noviembre, coincidiendo con la época de plena fructificación de la especie, por lo que se sugiere el transporte de los frutos alados de $M$. bahamensis por vientos huracanados, de la Península de Yucatán a las Islas Bahamas.

Esta especie no ha sido colectada en Cuba; aparentemente no crece en la Isla, a pesar de que algunas zonas costeras del este y sur de Cuba son de origen calizo (Anónimo, 1970), y de que la dirección de los ciclones indica que pudiera encontrarse ahí.

En el caso de la Península de Florida, que también está formada por sustrato calizo y que presenta algunas afinidades de su flora con la de Yucatán, las condiciones no parecen ser adecuadas para el establecimiento de esta especie, ya que tiene grandes áreas pantanosas.

Aún cuando no puede descartarse la posibilidad de que algún propágulo de esta especie haya llegado a Cuba o a Florida, existen diversos factores que son determinantes para el establecimiento de cualquier especie, como son las condiciones del suelo invadido, la madurez, la viabilidad y la depredación de las semillas, así como la competencia con otras especies.

\section{LISTADOS}

Especies asociadas con $M$. bahamensis en diferentes tipos de vegetación primaria y secundaria de la parte mexicana de la Península de Yucatán:

LISTADO 1: Areas taladas dentro de las selvas bajas y medianas deciduas:

Acacia collinsii Safford, A. pennatula (Schlecht. \& Cham.)Benth., Bauhinia divaricata L., Caesalpinia gaumeri Greenm., Desmodium incanum Schinz \& Thelling, Diphysa carthagenensis Jacq., Piscidia piscipula (L.) Sarg., Pithecellobium albicans (Kunth) Benth., P. tortum Mart., Senna uniflora (Miller) I. et B., S. villosa (Miller) I. et 
B. y Zapoteca formosa (Kunth) H. Hern. subsp. formosa. Otras especies asociadas son: Bursera simaruba (L.) Sarg., Cecropia peltata L., Guettarda combsii Urban, Gymnopodium antigonoides (Rob.) Blake, Luehea speciosa Willd. y Malvaviscus arboreus Cav.

LISTADO 2: Claros y áreas taladas de la selva baja subdecidua con Pseudophoenix, Coccothrinax y Thrinax:

Acacia dolychostachya Blake, A. gaumeri Blake, Bursera simaruba (L.) Sarg., Caesalpinia gaumeri Greenm., Diphysa carthagenensis Jacq., Gymnopodium floribundum Rolfe, Helicteres baruensis Jacq., Piscidia piscipula (L.) Sarg., Pithecellobium keyensis Britton ex Coker, $P$. platylobum (Spreng) Urban, Randia aculeata L., Thevetia thevetioides (HBK) Schum. y Torrubia linearibracteata (Heimerl.) Standl.

LISTADO 3: Claros y sitios alterados de la selva de transición entre baja y mediana subdecidua:

Acacia dolychostachya Blake, A. gaumeri Blake, Achatocarpus nigricans Triana, Alseis yucatanensis Standl., Alvaradoa amorphoides Liebm., Aphelandra deppeana Schlecht. \& Cham., Arrabidaea floribunda (H.B.K.) Loes, Bauhinia divaricata L., B. jenningsii Willson, Caesalpinia gaumeri Greenm., Erythrina standleyana Krukoff, Hampea trilobata Standl., Lonchocarpus castilloi Standl., Lysiloma latisiliqua (L.) Benth., Mimosa pudica L., Neea choriophylla Standl., Neea choriophylla (Tress) Blake, Securidaca silvestris Schlecht. y Zapoteca formosa (Kunth) H. Hern. subsp. formosa.

LISTADO 4: Areas perturbadas de la selva alta subperennifolia con Manilkara y Thrinax:

Acacia gaumeri Blake, A. globulifera Safford., A. pennatula (Schlecht. \& Cham.) Benth., Caesalpinia mollis Kunth, Coursetia caribaea (Jacq.) Lavin, Heliocarpus donellsmithii Rose, H. mexicanus (Turez.) Sprague, Lonchocarpus punctatus Kunth, Mimosa pudica L., Pithecellobium albicans (Kunth) Benth., destacando algunos elementos primarios como Manilkara zapota (L.) Royen, Sabal yapa Wright, Thrinax radiata Lodd. ex J.A. \& J.H. Schult. y Zapoteca formosa (Kunth) H. Hern. subsp. formosa .

LISTADO 5: Areas quemadas y taladas de la selva alta perennifolia con Manilkara, Thrinax y Coccothrinax:

Acacia dolychostachya Blake, A. gaumeri Blake, Cecropia obtusifolia Bert., C. peltata L., Lysiloma latisiliqua (L.) Benth. y Sabal yapa Wright.

LISTADO 6: Areas perturbadas de la selva alta subperennifolia con Manilkara, Bucida y Crysophila:

Alvaradoa amorphoides Liebm., Arrabidaea floribunda (H.B.K.) Loes, Bauhinia herrerae (Britton \& Rose) Standl. \& Steyerm., Bucida buceras L., Cecropia obtusifolia Bert., C. peltata L., Dalbergia glabra (Mill.) Standl., Diphysa carthagenensis Jacq., Lysiloma latisiliqua (L.) Benth., Mimosa pudica L., Pisonia aculeata L., Spondias mombin L., Swietenia macrophylla y Trema micrantha (L.) Blume, entre otras. 
LISTADO 7: Selvas del sur de Quintana Roo:

Acacia angustissima (Mill.) Kuntze, Brosimum alicastrum Swartz, Bursera simaruba (L.) Sarg., Chrysophyllum mexicanum Brand., Distietis laxiflora (DC.) Greenm., Lonchocarpus castilloi Standl., Luehea speciosa Willd., Mimosa albida H. \& B. ex Willd., Sabal mauritiiformis Griseb. \& Wendl., Thevetia ahouai (L.) A. DC. y Tournefortia maculata Jacq.

LISTADO 8: Tintales alterados:

Achatocarpus nigricans Triana, Bucida buceras L., Caesalpinia yucatanensis Greenm., Coccoloba cozumelensis Hemsl., Croton reflexifolius H.B.K., Dalbergia glabra (Mill.) Standl., Erythroxylon areolatum L., Eugenia lundellii Standl., Hampea trilobata Standl., Hyperbaena winzerlingii Standl., Jatropha gaumeri Greenm., Mimosa pigra L. y Sebastiania adenophora Pax. \& Hoffm.

LISTADO 9: Bajos alterados:

Bucida buceras L., Cameraria latifolia L., Chrysobalanus icaco L., Chrysophyllum mexicanum Brand., Croton reflexifolius H.B.K., Dalbergia glabra (Mill.) Standl., Diphysa carthagenensis Jacq., Erythroxylon areolatum L., Metopium brownei (Jacq.) Urban y Myrica cerifera $\mathrm{L}$.

LISTADO 10: Terrenos de cultivo abandonados o en barbecho:

Acacia angustissima (Mill.) Kuntze, A. gaumeri Blake, Bursera simaruba (L.) Sarg, Croton flavens L., C. rhamnifolius H.B.K., Dalbergia glabra (Mill.) Standl., Dioscorea alata L., Erythroxylon brevipes DC., Mimosa pudica L., Stizolobium pruriens (L.) Madikus, Gymnopodium floribundum Rolfe, G. antigonioides (Rob.) Blake, Heliotropium angiospermun Murr, Neomillspaughia emarginata (Gross) Blake, Pithecellobium albicans (Kunth) Benth., Rhynchosia minima (L.) DC., Ruellia tuberosa L., Sclerocarpus divaricatus (Benth. \& Hook.) Hemsl., Sida acuta Burm., Solanum verbascifolium L. y Viguiera dentata var. helianthoides (Cav.) Sprengel.

LISTADO 11: Orilla de caminos:

Acacia angustissima (Mill.) Kuntze, $A$. dolichostachya Blake, $A$. gaumeri Blake, $A$. glauca (L.) Moench, Bauhinia divaricata L., B. ungulata L., Dalbergia glabra (Mill.) Standl., Leucaena leucocephala (Lam.) Dewit, Lysiloma latisiliqua (L.) Benth., Passiflora foetida L., Piscidia piscipula (L.) Sarg. y Tephrosia cinerea (L.) Pers.

AGRADECIMIENTOS. Este trabajo ha sido financiado en parte por el CONACyT, Convenios: PCECCNA-050745, D112-903774 y PCECBDA-000914; y por la DGICSA de la Secretaría de Educación Pública, Convenio: 91-09-002-39. 


\section{LITERATURA CITADA}

ANÓNIMO. 1970. Atlas Nacional de Cuba. Instituto de Geografía de la Academia de Ciencias de Cuba e Instituto de Geografía de la Academia de Ciencias de la URSS. La Habana, Cuba.

BARRERA, M.A., A. BARRERA V. y R.M. LópEZ FrANCO. 1976.Nomenclatura etnobotánica maya. Una interpretación taxonómica. Centro Regional del Sureste 36. INAH, SEP. México.

BARTTLET, H.H. 1936. Botany of the maya area I. A method of procedure for field work in tropical american phytogeography based upon a botanical reconnaissance in parts of British Honduras and the Peten forest of Guatemala. Carnegie Inst. Wash. Publ. 461:3-25.

BENTHAM, G. 1842. Notes on Mimoseae, with a short synopsis of species. J. Bot. (Hooker) 4:408.

BRITTON, N.L. 1928. Pteromimosa. North Amer. Fl. 23:171-172.

GRETHER, R. 1982. Aspectos ecológicos de Mimosa biuncifera y Mimosa monancistra en el noroeste del estado de Guanajuato. Bol. Soc. Bot. México 43:43-60.

GUN, C.R.y J.V. DENNIS. 1976. World guide to tropical drift seeds and fruits. Ed. Demeter Press, New York. 240p.

HERNÁNDEZ X., E. 1962. Los recursos naturales del sureste y su aprovechamiento. La Agricultura. Revista Chapingo (Ser. 2) 2 (6):1-63.

LUNDELL, C.L. 1934. Preliminary sketch of the phytogeography of the Yucatan Peninsula. Carnegie Inst. Wash. Publ. 436:257-321.

LUNDELL, C.L.1937.The vegetation of Peten. Carnegie Inst. Wash. Publ. 478:1-244.

MiRANDA, F. 1964: Vegetación de la Península Yucateca. Sobretiros del Colegio de Postgraduados, Chapingo, México.

QUERO, H.J. 1981. Pseudophoenix sargentii in the Yucatan Peninsula, Mexico. Principes 25:63-72.

Rose, J.N. y B.L. ROBINSON. 1903. Studies of Mexican and Central American plants.New species of Acacia, Mimosa and Pithecellobium. Contr. U.S. Natl. Herb. 8:32-33.

RUDD, V.E. 1969. Mimosa bahamensis a Bahama-Yucatan disjunct. Phytologia 18:143-146.

SouZA-Novelo, N. 1945. Apuntes relativos a la flora de Yucatán. Mérida, Yucatán, México.

SouzA-Novelo, N., V.M. SuÁreZ-Molina y A. BARrerA-VÁZQUEZ. 1981. Plantas melíferas y poliníferas de Yucatán. Fondo Editorial de Yucatán, México.

STAGGERDA, M. 1941. Maya indians of Yucatan. Carnegie Inst. Wash. Publ. 531:1-280.

STANDLEY, P.C. 1930. Flora of Yucatan. Field. Mus. Nat. Hist. Publ. 279. Ser. Bot. 3:278-280.

TÉLlEZ, O. y M. SOUSA, 1982. Imágenes de la flora Quintanarroense. CICRO. 131p.

THOMPSON, J.D.1991. The biology of an invasiv plant. Bioscience 41:393-401. 